ARTICLE OPEN

\title{
Purely one-dimensional ferroelectricity and antiferroelectricity from van der Waals niobium oxide trihalides
}

\author{
Lei Zhang ${ }^{1,2}$, Cheng Tang ${ }^{1,2}$, Stefano Sanvito ${ }^{3}{ }^{3}$ and Aijun $\mathrm{Du}^{1,2 \bowtie}$
}

Intrinsic one-dimensional (1D) ferroelectric materials are rarely reported but are highly sought to break the size limit of nanostructured conventional ferroelectrics. Herein, we report a class of inborn $1 \mathrm{D}$ ferroelectric nanowires, namely $1 \mathrm{D} \mathrm{NbOX_{3 }}(\mathrm{X}=\mathrm{Cl}$, $\mathrm{Br}$, and I), that can be directly obtained from experimentally realized van der Waals crystals. In addition to the sizable spontaneous polarization, 1D $\mathrm{NbOX}_{3}$ exhibits low ferroelectric switching barriers, small coercive electric fields, and high critical temperature, governed by the hybridization of the Nb empty $d$ orbitals and the $\mathrm{O} p$ orbitals ( $d^{0}$ rule). Moreover, the double-channel structure of $1 \mathrm{D} \mathrm{NbOX}$ also enables the emergence of 1D antiferroelectric metastable states. Our findings not only propose a class of 1D ferroelectric materials toward the development of miniaturized and high-density electronic devices, but also pave an avenue of obtaining intrinsic 1D ferroelectrics from van der Waals crystals.

npj Computational Materials (2021)7:135; https://doi.org/10.1038/s41524-021-00602-9

\section{INTRODUCTION}

Ferroelectric (FE) materials sustaining a spontaneous electrical polarization that can be switched by an external electric field, have broad technological applications, such as non-volatile randomaccess memories ${ }^{1-3}$, field-effect transistors ${ }^{4,5}$, sensors ${ }^{6,7}$, and photovoltaics ${ }^{8,9}$. Driven by the increasing demand for device miniaturization, significant progress has been made in two/onedimensional (2D/1D) ferroelectrics. The exploration of 2D FE materials started with the direct downscaling of conventional bulk FE materials, a strategy found numerous obstacles such as enhanced depolarizing electrostatic fields, severe surface reconstruction, dangling bonds at the surface, etc., thus imposing great limits on their use for practical applications ${ }^{10-13}$. The recent emergence of van der Waals (vdW) layered 2D FE materials ${ }^{14,15}$ has stimulated extensive research, both experimentally and theoretically, by providing a possible solution to overcome these challenges thanks to their inborn atomic-thin structures ${ }^{16-27}$. Compared to 2D ferroelectrics, 1D FE materials are expected to be superior for building high-density FE devices. Several 1D FE nanostructures, including nanowires, nanotubes, nanoribbons, and belts, have been proposed. These are all based on manipulating conventional FE compounds ${ }^{28-33}$, meaning that such structures could suffer from the same drawbacks of their 2D counterparts. Moreover, the diameters of these 1D structures usually range from several tens to hundreds of nanometers, namely they are far from a nearly atom-scale size ${ }^{28,34}$. Ideally, 1D ferroelectrics should not only possess robust ferroelectricity but also simultaneously harbor small diameters, strong chemical bonding within the chain, and high stability. In order to overcome these challenges, it is highly important to explore new routes for the discovery of 1D ferroelectrics.

Experimentally a number of vdW crystals made of weakly bonded 1D building blocks (chains or molecular wires) have been successfully fabricated ${ }^{35-38}$. Inspired by the exfoliation of 2D functional materials from bulk layered structures, such type of vdW crystals could offer a great platform to explore the rich physics within the pure 1D limit. To date, research on 1D materials obtained from vdW bulk compounds is still limited, but some interesting properties have been already discussed, such as charge density waves in $\mathrm{NbSe}_{3}{ }^{39}$, nontrivial band topology in $\mathrm{TiCl}_{3}{ }^{40}$, and power-law dependent tunneling conductance in $\mathrm{MoSe}^{41}$. Importantly, the isolation of pure 1D structures from vdW crystals has been demonstrated possible in molybdenum based polyoxometalates $^{42}$. If the $1 \mathrm{D}$ building block in a vdW crystal possesses broken centrosymmetry along the chain direction, then intrinsic 1D ferroelectrics at ultrasmall dimensions could be obtained via exfoliation from the corresponding vdW crystals.

In this work we report a family of intrinsic 1D ferroelectrics, namely $1 \mathrm{D} \mathrm{NbOX}_{3}(\mathrm{X}=\mathrm{Cl}, \mathrm{Br}$, and I), which are analyzed by mean of density functional theory (DFT) and model Hamiltonian Monte Carlo (MC) simulations. 1D $\mathrm{NbOX}_{3}$ could be easily exfoliated from the experimentally synthesized vdW crystals due to the small binding energy. Moreover, $1 \mathrm{D} \mathrm{NbOX}{ }_{3}$ exhibits sizable spontaneous polarization above room temperature, low FE transition barriers, and low coercive electric fields. The two-channel 1D geometry of $\mathrm{NbOX}_{3}$ also enables the emergence of AFE metastable states, which has never been reported in the purely 1D regime. Our findings highlight an interesting avenue to realize intrinsic 1D ferroelectricity and antiferroelectricity from the vdW crystals.

\section{RESULTS AND DISCUSSION}

\section{Structural exploration and stability}

The structures of the experimental bulk phases ${ }^{43,44}$ of $\mathrm{NbOX}_{3}(\mathrm{X}=$ $\mathrm{Cl}, \mathrm{Br}$ and I) are presented in Fig. $1 \mathrm{a}-\mathrm{C}$. Bulk $\mathrm{NbOCl}_{3}$ and $\mathrm{NbOBr}_{3}$ crystalize in a tetragonal lattice with the $P \overline{4} 2{ }_{1} m$ space group, while $\mathrm{NbOl}_{3}$ displays a monoclinic structure with a lowered $\mathrm{C}_{2}$ symmetry. The PBE-calculated lattice parameters are summarized in Table 1 and they are in great agreement with the experimental values ${ }^{43,44}$. Interestingly, bulk $\mathrm{NbOX}_{3}$ possesses a peculiar unit cell consisting of two parallel $1 \mathrm{D} \mathrm{NbOX}$ nanowires, where the $\mathrm{Nb}$ ions show an off-center displacement, $d_{\mathrm{Nb}}$, along the O-Nb-O atomic chain direction. In the 3D crystals, $d_{\mathrm{Nb}}$ in neighboring 1D nanowires aligns antiparalleley in $\mathrm{NbOCl}_{3} / \mathrm{NbOBr}_{3}$, and parallely

${ }^{1}$ School of Chemistry and Physics, Queensland University of Technology, Brisbane, Australia. ${ }^{2}$ Centre for Materials Science, Queensland University of Technology, Brisbane, Australia. ${ }^{3}$ School of Physics and CRANN Institute, Trinity College, Dublin 2, Ireland. ${ }^{凶}$ email: aijun.du@qut.edu.au 

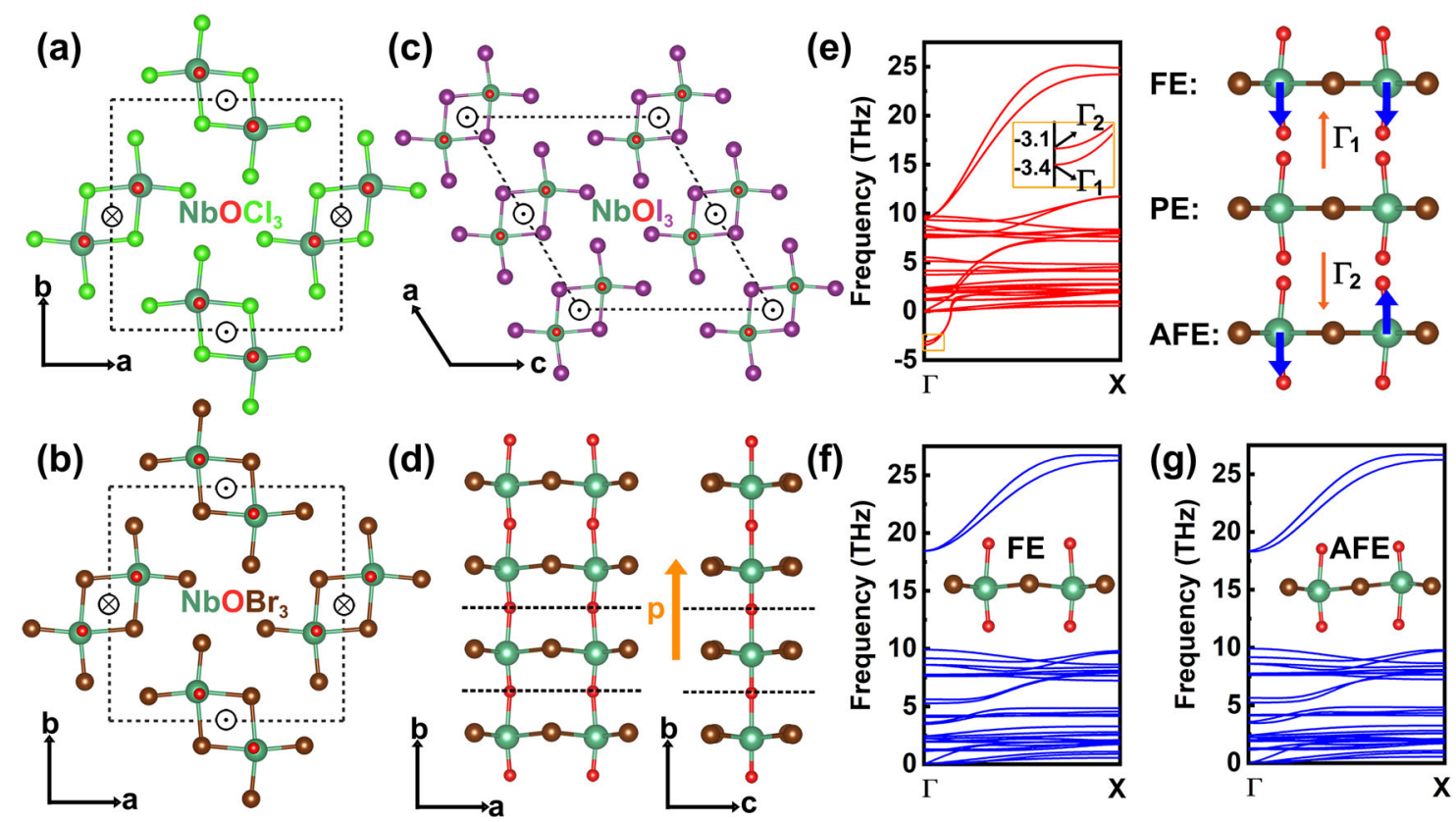

Fig. 1 Structrual configurations and the phonon spectra. a-c Crystal structures of the experimentally synthesized bulk phases of $\mathrm{NbOCl}_{3}$ $\mathrm{NbOBr}_{3}$, and $\mathrm{NbOl}_{3}$. The polarization direction of the individual 1D nanowires is explicitly shown by the dotted and crossed circles. $\mathbf{d}$ Atomic structure of $1 \mathrm{D} \mathrm{NbOBr}_{3}$. e Phonon spectrum of the PE phase of $1 \mathrm{D} \mathrm{NbOBr}_{3}$ and the eigenvectors of the soft-phonon modes at $\Gamma$ point. f, $\mathbf{g}$ Phonon spectra of the FE and AFE phases of $1 \mathrm{D} \mathrm{NbOBr}$.

Table 1. The lattice parameters $(a, b$, and $c)$, the $\mathrm{Nb}$ polar displacement $\left(d_{\mathrm{Nb}}\right)$ relative to the center of the $\mathrm{NbO}_{2} \mathrm{X}_{4}$ octahedra, and the spontaneous polarization $(P)$ for bulk and 1D $\mathrm{NbOX}_{3}$.

\begin{tabular}{|c|c|c|c|c|c|}
\hline & $a(\AA)$ & $b(\AA)$ & $c(\AA \AA)$ & $d_{\mathrm{Nb}}(\AA)$ & $P\left(\mu C\right.$ per $\left.\mathrm{cm}^{2}\right)$ \\
\hline \multicolumn{6}{|l|}{$\mathrm{NbOCl}_{3}$} \\
\hline Bulk & $10.97(0.6 \%)$ & $10.97(0.6 \%)$ & $3.92(0.7 \%)$ & 0.18 & $0.00(0.00)$ \\
\hline 1D AFE & $\mathrm{N} / \mathrm{A}$ & 3.92 & $\mathrm{~N} / \mathrm{A}$ & 0.18 & $0.00(0.00)$ \\
\hline \multicolumn{6}{|l|}{$\mathrm{NbOBr}_{3}$} \\
\hline 1D AFE & $\mathrm{N} / \mathrm{A}$ & 3.91 & $\mathrm{~N} / \mathrm{A}$ & 0.16 & $0.00(0.00)$ \\
\hline \multicolumn{6}{|l|}{$\mathrm{NbOl}_{3}$} \\
\hline Bulk & $14.76(0.9 \%)$ & $3.94(1.3 \%)$ & $12.74(1.1 \%)$ & 0.16 & $15.49(6.18)$ \\
\hline 1D FE & $\mathrm{N} / \mathrm{A}$ & 3.94 & $\mathrm{~N} / \mathrm{A}$ & 0.15 & $15.56(3.11)$ \\
\hline 1D AFE & $\mathrm{N} / \mathrm{A}$ & 3.94 & $\mathrm{~N} / \mathrm{A}$ & 0.15 & $0.00(0.00)$ \\
\hline
\end{tabular}

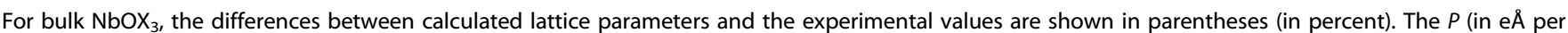
unit cell) is also given in parentheses for clarity. Note that bulk $\mathrm{NbOl}_{3}$ contains two $1 \mathrm{D}$ nanowires in its unit cell thus its $P$ (in e $\AA$ per unit cell) is around twice than that of $1 \mathrm{D} \mathrm{FE} \mathrm{NbOl}$.

in $\mathrm{NbOl}_{3}$, leading to anti-polarized and polarized bulk structures for $\mathrm{NbOCl}_{3} / \mathrm{NbOBr}_{3}$ and $\mathrm{NbOl}_{3}$, respectively.

Despite the different overall polar orders of bulk $\mathrm{NbOX}_{3}$, each 1D $\mathrm{NbOX}_{3}$ nanowire within the bulk phase is perfectly polarized and weakly vdW-bound. These features provide the opportunity to exfoliate the long-sought 1D polarized structures with perfect radical boundaries from experimentally accessible bulk phases. The binding energy $E_{\mathrm{b}}$ is then evaluated to determine the strength of the vdW interactions in the bulk. The relevant quantity is $E_{\mathrm{b}}=$ $E_{1 \mathrm{D}}-E_{\mathrm{bulk}} / 2$, where $E_{1 \mathrm{D}}$ and $E_{\mathrm{bulk}}$ are the energies of $1 \mathrm{D}$ and bulk $\mathrm{NbOX}_{3}$, respectively. The binding energies of $\mathrm{NbOCl}_{3}, \mathrm{NbOBr}_{3}$, and $\mathrm{NbOl}_{3}$ are calculated to be $62.2,78.0$, and $97.4 \mathrm{meV}$ per atom, respectively, and they are comparable to those of graphene $\left(52 \pm 5 \mathrm{meV} \text { per atom), } \mathrm{MoS}_{2} \text { (77 meV per atom }\right)^{45}$, and phosphorene $(81 \pm 5 \mathrm{meV} \text { per atom })^{46}$. This suggests that the extraction of $1 \mathrm{D} \mathrm{NbOX}$ from bulk is highly possible. The cohesive energy $E_{\mathrm{c}}$ is calculated by $E_{\mathrm{c}}=E_{1 \mathrm{D}}-2 E_{\mathrm{Nb}}-2 E_{\mathrm{O}}-6 E_{\mathrm{X}}$ where $E_{1 \mathrm{D}}, E_{\mathrm{Nb}}, E_{\mathrm{O}}$, and $E_{\mathrm{X}}$ represent the energy of $1 \mathrm{D} \mathrm{NbOX}_{3}$, single $\mathrm{Nb}, \mathrm{O}$, and $\mathrm{X}$ atoms, respectively. The $E_{\mathrm{c}}$ of $1 \mathrm{D} \mathrm{NbOCl}, \mathrm{NbOBr}_{3}$, and $\mathrm{NbOl}_{3}$ is calculated to be $-4.82,-4.46$, and $-4.09 \mathrm{eV} /$ atom, respectively, indicating their strong bonding characteristics.

1D $\mathrm{NbOX}_{3}$ possesses two parallel $\mathrm{NbOX}_{4}$ channels with the shared halogen atoms in the center as shown in Fig. 1d. In order to extensively explore the polar configurations possible in these double-channel 1D structures, the centrosymmetric phase $(P m m m$ symmetry) in which there is no $\mathrm{Nb}$ displacement along the $b$ axis 
is chosen as paraelectric (PE) configuration and the associated phonon spectra are examined first. Figure 1e presents the calculated phonon spectrum of 1D PE $\mathrm{NbOBr}_{3}$ as an example. Clearly, two optical phonon modes show imaginary frequency $\left(\Gamma_{1}=-3.4 \mathrm{THz}\right.$ and $\left.\Gamma_{2}=-3.1 \mathrm{THz}\right)$ at the $\Gamma$ point, indicating a dynamical instability of $1 \mathrm{D} P E \mathrm{NbOBr}_{3}$. Further analysis of the phonon eigenvectors confirms that the $\Gamma_{1}$ and $\Gamma_{2}$ soft modes correspond to the off-center displacement, $d_{\mathrm{Nb}}$, with either identical or opposite sign in the two $\mathrm{NbOBr}_{4}$ channels. Such soft modes spontaneously drive the system into either a FE (space group of Pmm2) or an AFE (space group of $P 2 / \mathrm{m}$ ) state, whose dynamical stabilities are confirmed by the phonon spectra (see Fig. 1f, g) showing no negative frequencies. Similarly, we can identify the stable FE and AFE phases of $1 \mathrm{D} \mathrm{NbOCl}$ and $\mathrm{NbOl}_{3}$, as shown in Supplementary Fig. 1. We also consider head-to-head antipolar configurations as shown in Supplementary Fig. 2, which are energetically not favorable compared with the FE and AFE phases. The elastic constants are calculated to be 76.93, 73.75, and 54.67 GPa for $1 \mathrm{D} \mathrm{FE} \mathrm{NbOCl}_{3}, \mathrm{NbOBr}_{3}$, and $\mathrm{NbOl}_{3}$ respectively by using density-functional perturbation theory (DFPT) method, suggesting their flexible 1D structures. The thermal stability of the ferroic states is confirmed by the small energy fluctuations and structural variations observed during $5 \mathrm{ps}$-long AIMD simulations at $300 \mathrm{~K}$ (see Supplementary Fig. 3). It is worth noting that the 1D AFE states are only slightly higher in energy $(<3 \mathrm{meV})$ than the FE ones, suggesting that metastable $1 \mathrm{D}$ AFE $\mathrm{NbOX}_{3}$ might also be experimentally accessible.

\section{Ferroelectric polarization and switching barrier}

The central symmetry of $1 \mathrm{D} \mathrm{FE} \mathrm{NbOX}_{3}$ is broken by the cooperative displacements of the $\mathrm{Nb}$ ions in both $\mathrm{NbOX}_{4}$ channels, leading to a significant spontaneous polarization, $P$, along the chain direction. The magnitude of $P$ is calculated by Berry phase through the modern theory of polarization ${ }^{47,48}$. The volume of $1 \mathrm{D} \mathrm{FE} \mathrm{NbOX}_{3}$ is estimated with the van der Waals interchain spacing of bulk $\mathrm{NbOX}_{3}$, analogusly to what done for 2D vdW ferroelectric materials. The polarizaiton of $1 \mathrm{D} \mathrm{FE} \mathrm{NbOCl}_{3}$,
$\mathrm{NbOBr}_{3}$, and $\mathrm{NbOl}_{3}$ is calculated to be $23.76,20.19$, and $15.56 \mu \mathrm{C}$ per $\mathrm{cm}^{2}$, respectively, values larger or comparable to those of many 2D FE materials, including $1 \mathrm{~T}$ transition-metal dichalcogenides $^{49}$, group IV monochalcogenides ${ }^{50,51}$, metal chalcogendiphosphates ${ }^{52,53}$, conventional bulk FE materials like $\mathrm{BaTiO}_{3}{ }^{54}$, and some lead-zirconate-titanate (PZT) ${ }^{55,56}$. The piezoelectric coefficients along the polar direction are calculated to be 2.69, 2.71, and $3.01 \mathrm{C}$ per $\mathrm{m}^{2}$ for $1 \mathrm{D} \mathrm{NbOCl}_{3}, \mathrm{NbOBr}_{3}$, and $\mathrm{NbOl}_{3}$ respectively by using DFPT method. In the $1 \mathrm{D}$ AFE phases, the $\mathrm{Nb}$ opposite polar displacements in the two $\mathrm{NbOX}_{4}$ channels cancel out, giving an overall vanishing $P$. Their polar strength can be estimated from the magnitudes of $d_{\mathrm{Nb}}$ in the $\mathrm{NbOX}_{4}$ channels, which are close to what found for the FE phases (see Table 1).

In a FE material, the direction of the electric polarization can be switched by an external electric field, hence it is of paramount importance to understand the kinetics of the polarization reversal process in $1 \mathrm{D} \mathrm{NbOX}_{3}$. In order to explore the most favorable transition pathway connecting the two energetically degenerate states with opposite polarization directions, we systematically scanned the energy surface of $1 \mathrm{D} \mathrm{NbOX}_{3}$ with respect to two independent reaction coordinates, $d_{\mathrm{Nb} 1}$ and $d_{\mathrm{Nb} 2}$, namely the polar displacements of $\mathrm{Nb}$ in the individual channels. As shown in Fig. 2a-c, two low-energy FE and AFE states with opposite polarized directions (namely, $\mathrm{S}_{\mathrm{FE}} / \mathrm{S}_{\mathrm{FE}}^{\prime}$ and $\mathrm{S}_{\mathrm{AFE}} / \mathrm{S}_{\mathrm{AFE}}^{\prime}$ ) are found along the diagonal directions, and they are separated by a highenergy PE state. This is consistent with the predicted two soft modes of $1 \mathrm{D} \mathrm{PE} \mathrm{NbOX}$, which spontaneously drive the PE state to a lower-energy state (either FE or AFE). FE switching would then proceed by crossing the AFE metastable state, instead of being a direct transition going through the PE configuration, due to the large energy difference between the FE and PE states. The NEB method is further exploited to refine the transition pathways from $S_{\mathrm{FE}}$ to $\mathrm{S}_{\mathrm{FE}}^{\prime}$ and to determine accurately the transition barriers. As shown in Fig. $2 \mathrm{~d}, d_{\mathrm{Nb}}$ in one of the $\mathrm{S}_{\mathrm{FE}}$ channels is first reversed, while that in another channel remains unchanged, leading the system to an intermediate AFE state. Then, $d_{\mathrm{Nb}}$ in the unchanged channel gets reversed as well, realizing the transition from $\mathrm{S}_{\mathrm{FE}}$
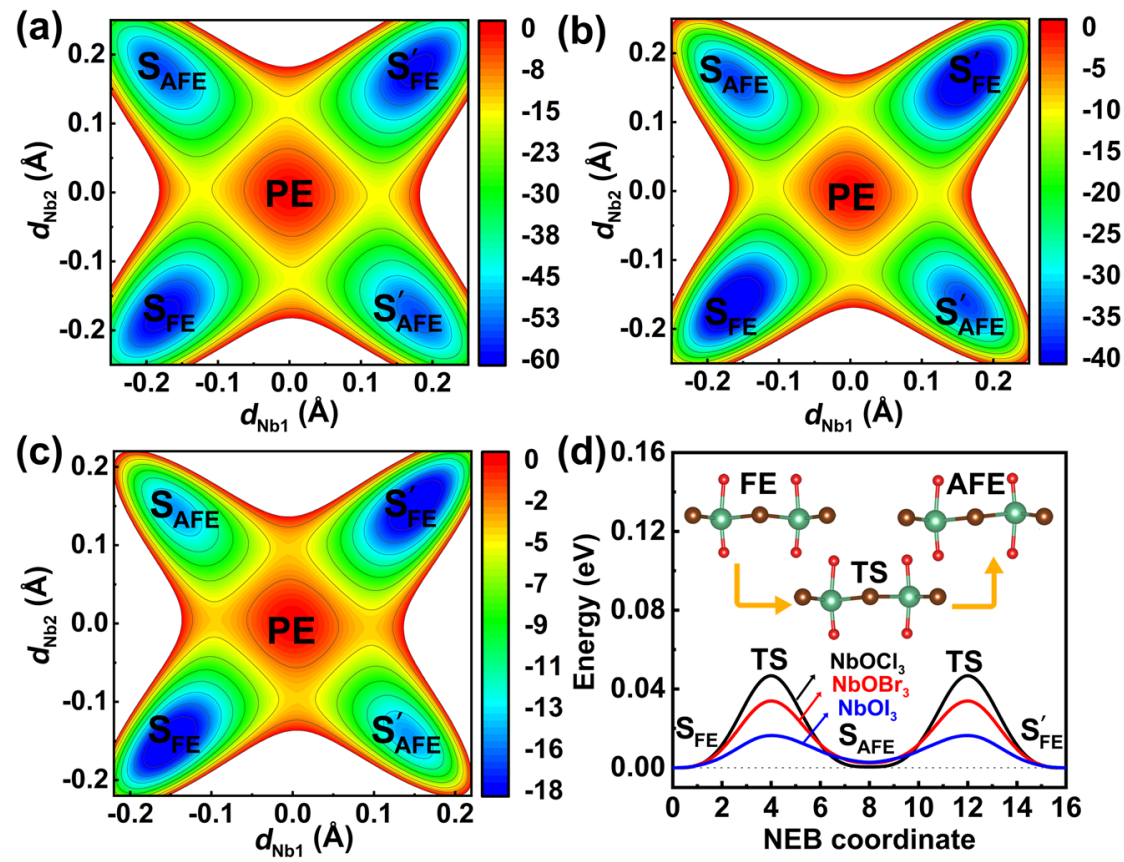

Fig. 2 Energy map and NEB results. a-c The energy contour plot (in meV) of the $1 \mathrm{D} \mathrm{NbOX}$ unit cell as a function of the polar displacements of two $\mathrm{Nb}$ ions for $\mathrm{NbOCl}_{3}, \mathrm{NbOBr}_{3}$, and $\mathrm{NbOl}_{3}$, respectively. The energy of the PE phases is set to zero. $\mathbf{d}$ Energy profiles along the $\mathrm{NEB}$ pathway for the polarization switching of $1 \mathrm{D} \mathrm{FE} \mathrm{NbOX}_{3}$. The inset shows the structure evolution from FE to AFE states through the transition state (TS). 

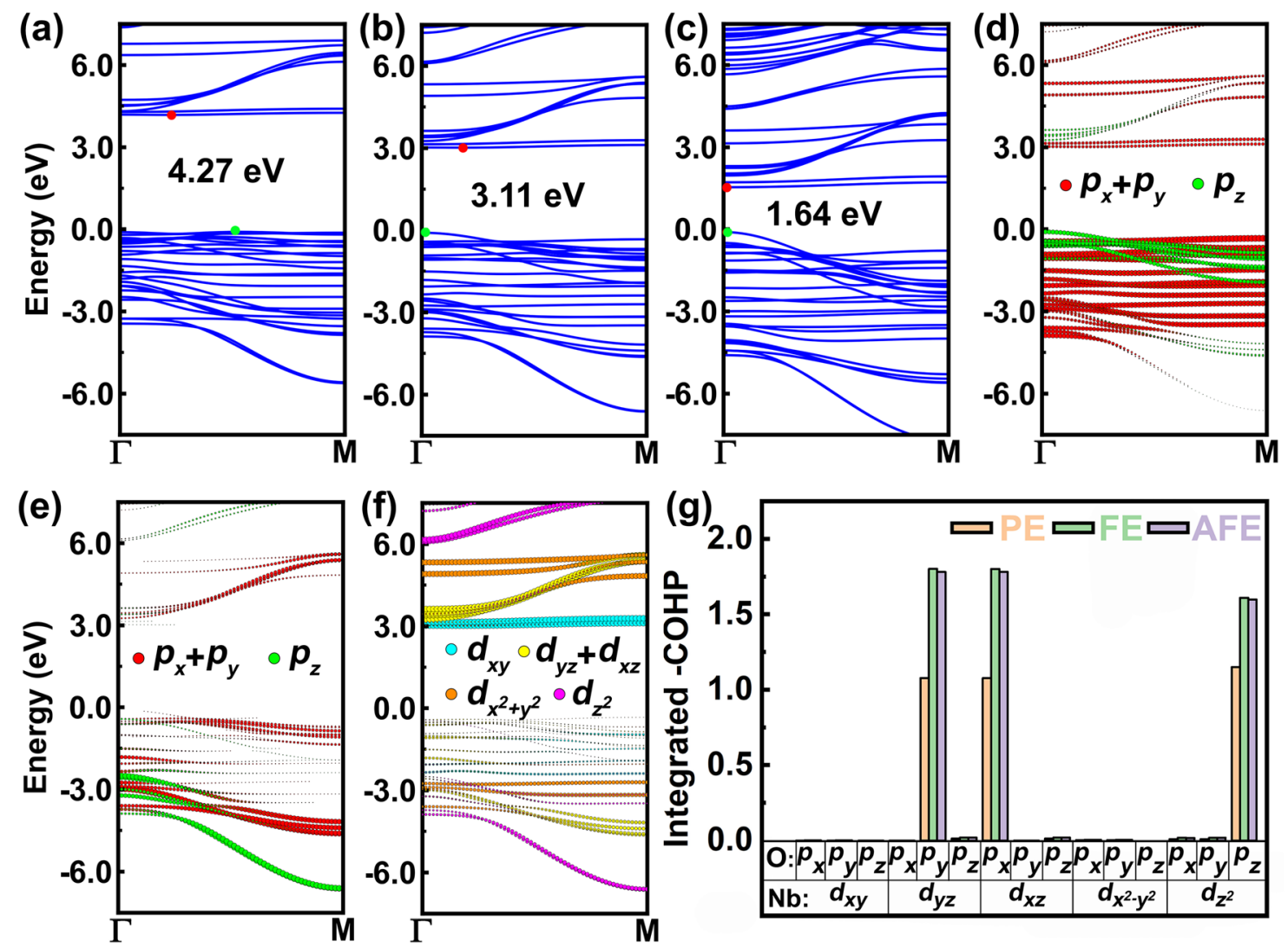

Fig. 3 Band structures and integrated -COHP. a-c The band structures of 1D ferroelectric $\mathrm{NbOCl}_{3}, \mathrm{NbOBr}_{3}$, and $\mathrm{NbOl}_{3}$, respectively. The conduction band minimum (CBM) and valence band maximum (VBM) are marked by red and green dots, respectively. $\mathbf{d}-\mathbf{f}$ The orbital-resolved band structures for $\mathrm{Br}, \mathrm{O}$, and $\mathrm{Nb}$ in $1 \mathrm{D} \mathrm{NbOBr}{ }_{3}$, respectively. The radii of the colored dots represent the contribution of the corresponding atomic orbitals. For clarity, the global axes are rotated to align with the $\mathrm{NbO}_{2} \mathrm{Br}_{4}$ octahedron, i.e., the $x / y$ and $\mathrm{z}$ axes are along the $\mathrm{Nb}-\mathrm{Br}$ and $\mathrm{Nb}-\mathrm{O}$ bonding directions, respectively. $\mathbf{g}$ The integrated -COHP for all the $d-p$ interactions between Nb and O atoms in PE, FE, and AFE phases of $1 \mathrm{D} \mathrm{NbOBr}$.

to $\mathrm{S}_{\mathrm{FE}}^{\prime}$. The overall transition barriers are calculated to be only 47 , 34 , and 16 meV for $1 \mathrm{D} \mathrm{FE} \mathrm{NbOCl}_{3}, \mathrm{NbOBr}_{3}$, and $\mathrm{NbOl}_{3}$ respectively. These values are much smaller than those of many widely studied FE materials, such as $\mathrm{PbTiO}_{3}, \mathrm{BaTiO}_{3}$, and $2 \mathrm{D} \ln _{2} \mathrm{Se}_{3}{ }^{57-59}$.

\section{Electronic properties}

Having explored the polar nature of $1 \mathrm{D} \mathrm{NbOX}_{3}$, we further investigate the origin of the 1D ferroelectricity and antiferroelectricity. $1 \mathrm{D} \mathrm{NbOX}$ nanowires are found to be semiconductors with band gaps ranging between $1.4 \mathrm{eV}$ and $4.3 \mathrm{eV}$ and display similar band compositions, as shown in Fig. 3a-f and Supplementary Figs. 4-6. By taking $1 \mathrm{D} \mathrm{FE} \mathrm{NbOBr}_{3}$ as an example, we plot the orbitalresolved band structures for $\mathrm{Br}, \mathrm{O}$, and $\mathrm{Nb}$ atoms in $1 \mathrm{D} \mathrm{NbOBr}_{3}$ (see Fig. $3 \mathrm{~d}-\mathrm{f}$ ). The $\mathrm{Nb}-d_{\mathrm{x}}{ }^{2}-\mathrm{y}{ }^{2} / \mathrm{Br}-p_{\mathrm{x} / \mathrm{y}}, \mathrm{Nb}-d_{\mathrm{xz} / \mathrm{yz}} / \mathrm{O}-p_{\mathrm{x} / \mathrm{y}}$, and $\mathrm{Nb}-\mathrm{d}_{\mathrm{z}}{ }^{2} /$ $\mathrm{O}-p_{\mathrm{z}}$ hybridization can be clearly seen below the Fermi level and it is expected from the $\mathrm{Nb}-\mathrm{Br}$ and $\mathrm{Nb}-\mathrm{O}$ bonding geometry. The mixing of $\mathrm{Nb}^{5+}$ empty $d$ orbitals and $\mathrm{O}^{2-} p$ orbitals along the polar direction thus dominates the emergence of the FE/AFE states in 1D $\mathrm{NbOBr}_{3}$, namely the $d^{0}$ principle is found for this class of compounds, in analogy with the well-known FE perovskite oxides $^{60,61}$. Figure $3 \mathrm{~g}$ and Supplementary Fig. 7 present the calculated -COHP (crystal orbital Hamilton population) integral for $1 \mathrm{D} \mathrm{NbOBr} 3$ and $\mathrm{NbOCl}_{3} / \mathrm{NbOl}_{3}$, which further confirms that the FE/ AFE states are stabilized by the enhanced orbital hybridization of $\mathrm{Nb}-d_{\mathrm{xz} / \mathrm{yz}} / \mathrm{O}-p_{\mathrm{x} / \mathrm{y}}$ and $\mathrm{Nb}-d_{\mathrm{z}}{ }^{2} / \mathrm{O}-p_{\mathrm{z}}$. Furthermore, we find the $p d \pi$ interactions of $\mathrm{Nb}-d_{\mathrm{xz} / \mathrm{yz}} / \mathrm{O}-p_{\mathrm{x} / \mathrm{y}}$ to show more prominent enhancements than the $p d \sigma$ interaction of Nb- $d_{\mathrm{z}}^{2} / \mathrm{O}-p_{\mathrm{z}}$. This is a signature also found for the O-Ti interactions in $\mathrm{BaTiO}_{3}$ and $\mathrm{PbTiO}_{3}{ }^{62}$.

\section{Monte Carlo simulations}

For practical applications, the critical temperature, $T_{C}$, of $1 \mathrm{D} \mathrm{FE}$ $\mathrm{NbOX}_{3}$ needs to be high enough so that their polarization can persist above room temperature. The effect of finite temperature is then investigated via Monte Carlo (MC) simulations based on a Landau-Ginzburg model ${ }^{20}$, in which the energy, $E$, of $1 \mathrm{D} \mathrm{NbOX}$ can be expressed by an expansion of the order parameter $d_{\mathrm{i}}\left(d_{\mathrm{Nb}}\right.$ of ith $\mathrm{NbO}_{2} \mathrm{Br}_{4}$ octahedron),

$$
E=\sum_{i}\left[\frac{A}{2} d_{i}^{2}+\frac{B}{4} d_{i}^{4}+\frac{C}{6} d_{i}^{6}\right]+\sum_{\langle i, j\rangle_{x}} \frac{D_{x}}{2}\left(d_{i}-d_{j}\right)^{2}+\sum_{\langle i, j\rangle_{y}} \frac{D_{y}}{2}\left(d_{i}-d_{j}\right)^{2}
$$

where the first term describes the on-site potential energy and the parameters $A, B$, and $C$ can be obtained by fitting the energy- $d_{\mathrm{Nb}}$ double-well curve (see Fig. 4a). The other two terms represent the harmonic interactions between two neighboring dipole moments (Supplementary Fig. 8) along the $x$ and $y$ directions, respectively. The coefficients $D_{\mathrm{x}}$ and $D_{\mathrm{y}}$ can be estimated based on the nearestneighbor approximation as shown in Supplementary Fig. 9. All the parameters $\left(A, B, C, D_{x}\right.$, and $\left.D_{y}\right)$ for $1 \mathrm{D} \mathrm{FE} \mathrm{NbOX}_{3}$ are summarized in Supplementary Table 1. The limitation of this model is discussed in Supplementary Note 1.

With the above effective Hamiltonian, Eq. (1), the phase transition at finite temperature can be investigated by $M C$ simulations. Figure $4 \mathrm{~b}$ plots the averaged polar displacements of $\mathrm{Nb}$ ions as a function of temperature. Clearly, this quantity drops abruptly at a temperature close to the $T_{C}$, suggesting the occurrence of a phase transition. The $T_{C}$ are then calculated from the singular point of the specific heat (see Supplementary Fig. 10) 
(a)

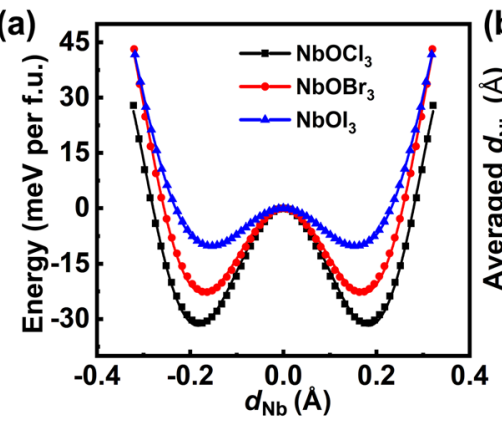

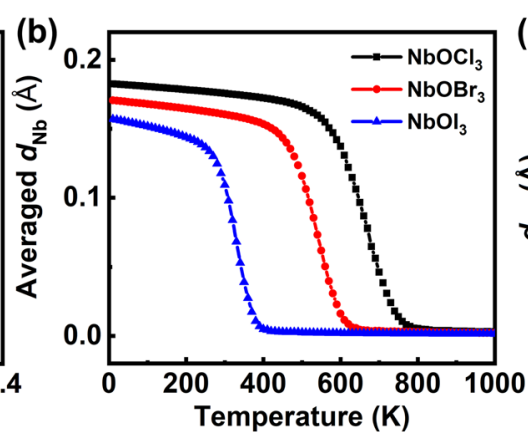

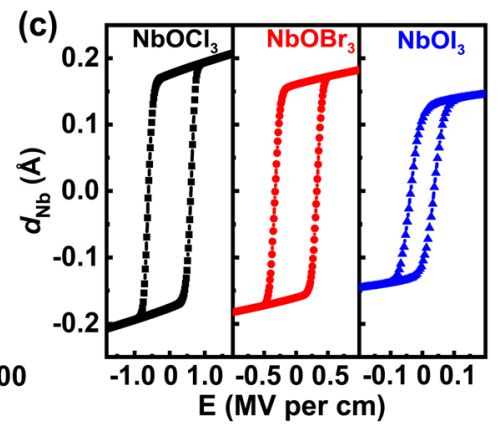

Fig. 4 Double-well potential and MC simulation results. a Energy- $d_{\mathrm{Nb}}$ double-well plots connecting the PE and the two degenerate FE phases ( $-P$ and $P$ states) in 1D NbOX . The DFT data is fitted using the Landau-Ginzburg model. $\mathbf{b}$ The averaged Nb polar displacements of $1 \mathrm{D}$ $\mathrm{FE} \mathrm{NbOX}$ as a function of temperature as obtained from MC simulations. c The ferroelectric hysteresis loops of $1 \mathrm{D} \mathrm{FE} \mathrm{NbOX}$ simulated by MC calculations at room temperature.

to be 630,520 , and $320 \mathrm{~K}$ for $\mathrm{NbOCl}_{3}, \mathrm{NbOBr}_{3}$, and $\mathrm{NbOl}_{3}$, respectively. The robust room-temperature ferroelectricity for $1 \mathrm{D}$ $\mathrm{NbOX}_{3}$ can also be validated by the averaged $d_{\mathrm{Nb}}$ during the last 2 ps in our AIMD simulations at $300 \mathrm{~K}$ (Supplementary Fig. 11), where no significant reduction of $d_{\mathrm{Nb}}$ is observed under thermal perturbation. Notably, the Tc of $1 \mathrm{D} \mathrm{FE} \mathrm{NbOX}_{3}$ follows the $\mathrm{NbOCl}_{3}>$ $\mathrm{NbOBr}_{3}>\mathrm{NbOl}_{3}$ order, mirroring the order of their double-well potential depths.

Finally, we investigate the electric field induced FE transition by MC simulations. When an electric field, E, is applied along the polar direction, an additional energy term $-\mathbf{E}\left(\Sigma d_{i} Z_{i}^{*}\right)$ must be incorporated into Eq. (1), where $Z_{i}^{*}$ is the Born effective charge. Figure $4 \mathrm{c}$ presents the simulated FE hysteresis loops. The critical electric fields that trigger the depolarization are determined to be around $0.61,0.33$, and $0.04 \mathrm{MV}$ per $\mathrm{cm}$ for $\mathrm{NbOCl}_{3}, \mathrm{NbOBr}_{3}$, and $\mathrm{NbOl}_{3}$, respectively, which are comparable or even smaller than those of reported FE materials including $\mathrm{HfO}_{2}(\sim 1 \mathrm{MV} \text { per } \mathrm{cm})^{63}$, $\mathrm{HfZrO}_{4}(\sim 1.2 \mathrm{MV} \text { per } \mathrm{cm})^{64}, \mathrm{CulnP}_{2} \mathrm{~S}_{6}(\sim 10 \mathrm{MV} \text { per } \mathrm{cm})^{65}$, and $2 \mathrm{D}$ $\mathrm{In}_{2} \mathrm{Se}_{3}(6-10 \mathrm{MV} \text { per } \mathrm{cm})^{66}$. The small coercive fields of 1D FE $\mathrm{NbOX}_{3}$ will substantially facilitate the FE switching under low electric voltage, promising great potential for low energy-cost FE devices.

In summary, we have predicted the long-sought intrinsic 1D ferroelectricity in $1 \mathrm{D} \mathrm{NbOX}$ nanowires. $1 \mathrm{D} \mathrm{NbOX}$ is highly likely to be exfoliated from the experimentally synthesized vdW bulk phases due to the small binding energies. Notably, 1D $\mathrm{NbOX}_{3}$ possesses great dynamical and thermal stabilities, sizable spontaneous polarizations, low switching barriers and coercive fields, and above room temperature $T c$, holding great potentials for applications in nanoscale FE devices such as high-density nonvolatile memories. In addition, the double-channel 1D structure also enables AFE metastable states in $1 \mathrm{D} \mathrm{NbOX}$, offering a great platform to explore complex ferroic orders down to the 1D limit. The polarized nature of $1 \mathrm{D} \mathrm{NbOX}_{3}$ originates from the $d^{0}$ rule, namely from the hybridization of the Nb- $d_{\mathrm{xz} / \mathrm{yz}} / \mathrm{O}-p_{\mathrm{x} / \mathrm{y}}$ and $\mathrm{Nb}-\mathrm{d}_{\mathrm{z}}{ }^{2} /$ O- $p_{z}$ orbitals, a mechanism similar to that found in conventional $\mathrm{FE}$ materials, like $\mathrm{PbTiO}_{3}$ and $\mathrm{BaTiO}_{3}$. Our findings highlight a class of intrinsic 1D FE compounds with extraordinary ferroelectricity and point to a feasible route for the exploration of exotic 1D physics from the vdW crystals containing 1D building blocks.

\section{METHODS}

\section{Geometry optimization and electronic structure calculations}

Our DFT calculations were carried out using the Vienna ab initio simulation package (VASP) ${ }^{67-69}$. The electron-core interaction was described by using the projector augmented wave (PAW) method ${ }^{70}$ and the exchange and correlation energy was treated with the generalized gradient approximation (GGA) parameterized by Perdew, Burke, and Ernzerhof (PBE) ${ }^{71,72}$. The $v d W$ interactions were included throught the DFT-D3 scheme ${ }^{73}$. The cutoff energy for the plane-wave expansion was set to $520 \mathrm{eV}$ and the Brillouin zone was sampled by a $1 \times 12 \times 1$ mesh. The geometries were fully optimized until the residual forces and the energy were converged to $0.005 \mathrm{eV}$ per $\AA$ and $1 \times 10^{-6} \mathrm{eV}$, respectively. A vacuum region always $>15 \AA$ was introduced to avoid the interaction between the neighboring periodic images. The Heyd-Scuseria-Ernzerhof hybrid functional (HSE06) was also utilized to obtain an accurate description of the electronic properties $^{74}$.

\section{Phonon, NEB, and AIMD calculations}

Phonon spectra were calculated based on $1 \times 4 \times 1$ supercells by using the finite displacement method as implemented in the PHONOPY code ${ }^{75}$. The threshold for energy is tightened to $10^{-9} \mathrm{eV}$ to get the accurate forces. The nudged elastic band (NEB) method ${ }^{76}$ was applied to study the FE phase transition, with 17 images used in total. Ab initio molecular dynamics (AIMD) simulations were carried out using $1 \times 8 \times 1$ supercells for a total of $5 \mathrm{ps}$ with a time step of $1.0 \mathrm{fs}$. The NVT ensemble is used in the simulations with the temperature controled by the Langevin thermostat ${ }^{77,78}$.

\section{Monte Carlo simulations}

Metropolis-algorithm MC simulations were performed for a periodic 1D supercell containing 20,000 unit cells. The first $2 \times 10^{8}$ MC steps were used for equilibration, followed by additional $2 \times 10^{8} \mathrm{MC}$ steps to obtain the thermal averages. Each simulation was repeated 100 times and then the results were averaged to eliminate numerical errors.

\section{DATA AVAILABILITY}

The data that support the findings of this study are available from the corresponding author upon reasonable request.

Received: 2 June 2021; Accepted: 28 July 2021; Published online: 19 August 2021

\section{REFERENCES}

1. Cao, Q. et al. Nonvolatile multistates memories for high-density data storage. ACS Appl. Mater. Interfaces 12, 42449-42471 (2020).

2. Chanthbouala, A. et al. Solid-state memories based on ferroelectric tunnel junctions. Nat. Nanotechnol. 7, 101-104 (2012).

3. Das, S. \& Appenzeller, J. F. An organic ferroelectric material based novel random access memory cell. Nano Lett. 11, 4003-4007 (2011).

4. Hoffman, J. et al. Ferroelectric field effect transistors for memory applications. Adv. Mater. 22, 2957-2961 (2010).

5. Khan, A. I., Keshavarzi, A. \& Datta, S. The future of ferroelectric field-effect transistor technology. Nat. Electron. 3, 588-597 (2020).

6. Lee, Y. et al. Flexible ferroelectric sensors with ultrahigh pressure sensitivity and linear response over exceptionally broad pressure range. ACS Nano 12, 4045-4054 (2018).

7. Wang, L., Teleki, A., Pratsinis, S. E. \& Gouma, P. I. Ferroelectric WO3 nanoparticles for acetone selective detection. Chem. Mater. 20, 4794-4796 (2008).

8. Yang, S. Y. et al. Above-bandgap voltages from ferroelectric photovoltaic devices. Nat. Nanotechnol. 5, 143-147 (2010). 
9. Yuan, Y., Xiao, Z., Yang, B. \& Huang, J. Arising applications of ferroelectric materials in photovoltaic devices. J. Mater. Chem. A 2, 6027-6041 (2014).

10. Han, H., Kim, Y., Alexe, M., Hesse, D. \& Lee, W. Nanostructured ferroelectrics: fabrication and structure-property relations. Adv. Mater. 23, 4599-4613 (2011).

11. Batra, I. P., Wurfel, P. \& Silverman, B. D. Phase transition, stability, and depolarization field in ferroelectric thin films. Phys. Rev. B 8, 3257-3265 (1973).

12. Sepliarsky, M., Stachiotti, M. G. \& Migoni, R. L. Surface reconstruction and ferroelectricity in PbTiO3 thin films. Phys. Rev. B 72, 014110 (2005).

13. Setter, N. et al. Ferroelectric thin films: review of materials, properties, and applications. J. Appl. Phys. 100, 051606 (2006).

14. Guan, Z. et al. Recent progress in two-dimensional ferroelectric materials. Adv. Electron. Mater. 6, 1900818 (2020).

15. Wu, M. \& Jena, P. The rise of two-dimensional van der Waals ferroelectrics. Wiley Interdiscip. Rev. Comput. Mol. Sci. 8, e1365 (2018).

16. Belianinov, A. et al. CulnP2S6 room temperature layered ferroelectric. Nano Lett. 15, 3808-3814 (2015).

17. Cui, C., Xue, F., Hu, W.-J. \& Li, L.-J. Two-dimensional materials with piezoelectric and ferroelectric functionalities. NPJ 2D Mater. Appl. 2, 18 (2018).

18. Higashitarumizu, N. et al. Purely in-plane ferroelectricity in monolayer SnS at room temperature. Nat. Commun. 11, 2428 (2020).

19. Zhang, L., Tang, C., Zhang, C. \& Du, A. First-principles screening of novel ferroelectric mxene phases with a large piezoelectric response and unusual auxeticity. Nanoscale 12, 21291-21298 (2020).

20. Fei, R., Kang, W. \& Yang, L. Ferroelectricity and phase transitions in monolayer group-IV monochalcogenides. Phys. Rev. Lett. 117, 097601 (2016).

21. Zhang, J.-J. et al. Type-Il multiferroic Hf2VC2F2 mxene monolayer with high transition temperature. J. Am. Chem. Soc. 140, 9768-9773 (2018).

22. Yang, Q., Xiong, W., Zhu, L., Gao, G. \& Wu, M. Chemically functionalized phosphorene: two-dimensional multiferroics with vertical polarization and mobile magnetism. J. Am. Chem. Soc. 139, 11506-11512 (2017).

23. Li, L. \& Wu, M. Binary compound bilayer and multilayer with vertical polarizations: two-dimensional ferroelectrics, multiferroics, and nanogenerators. ACS Nano 11, 6382-6388 (2017).

24. Ma, L., Jia, Y., Ducharme, S., Wang, J. \& Zeng, X. C. Diisopropylammonium bromide based two-dimensional ferroelectric monolayer molecular crystal with large in-plane spontaneous polarization. J. Am. Chem. Soc. 141, 1452-1456 (2019).

25. Huang, $C$. et al. Prediction of intrinsic ferromagnetic ferroelectricity in a transitionmetal halide monolayer. Phys. Rev. Lett. 120, 147601 (2018).

26. $\mathrm{Hu}, \mathrm{T}$. \& Kan, E. Progress and prospects in low-dimensional multiferroic materials. Wiley Interdiscip. Rev. Comput. Mol. Sci. 9, e1409 (2019).

27. $\mathrm{Xu}, \mathrm{C}$. et al. Electric-field switching of magnetic topological charge in type-I multiferroics. Phys. Rev. Lett. 125, 037203 (2020).

28. Rørvik, P. M., Grande, T. \& Einarsrud, M.-A. One-dimensional nanostructures of ferroelectric perovskites. Adv. Mater. 23, 4007-4034 (2011).

29. Hu, Y. et al. A chiral switchable photovoltaic ferroelectric 1D perovskite. Sci. Adv. 6, eaay4213 (2020).

30. Jung, J. H. et al. Lead-free $\mathrm{KNbO} 3$ ferroelectric nanorod based flexible nanogenerators and capacitors. Nanotechnology 23, 375401 (2012).

31. Kim, S. et al. Synthesis of monoclinic potassium niobate nanowires that are stable at room temperature. J. Am. Chem. Soc. 135, 6-9 (2013).

32. Mao, Y., Banerjee, S. \& Wong, S. S. Hydrothermal synthesis of perovskite nanotubes. Chem. Commun. 9, 408-409 (2003).

33. Kim, J. et al. Ferroelectricity in highly ordered arrays of ultra-thin-walled $\mathrm{Pb}(\mathrm{Zr}, \mathrm{Ti})$ O3 nanotubes composed of nanometer-sized perovskite crystallites. Nano Lett. 8, 1813-1818 (2008).

34. Liang, L., Kang, X., Sang, Y. \& Liu, H. One-dimensional ferroelectric nanostructures: synthesis, properties, and applications. Adv. Sci. 3, 1500358 (2016).

35. Cheon, G. et al. Data mining for new two- and one-dimensional weakly bonded solids and lattice-commensurate heterostructures. Nano Lett. 17, 1915-1923 (2017).

36. Stephens, J. \& Cruickshank, D. The crystal structure of $(\mathrm{Cro3}) \infty$. Acta Crystallogr. B Struct. Cryst. Cryst. Chem. 26, 222-226 (1970).

37. Shorafa, H. \& Seppelt, K. The structures of CrF5 and CrF5.SbF5. Z. Anorg. Allg. Chem. 635, 112-114 (2009).

38. Oeckler, O. \& Simon, A. Redetermination of the crystal structure of copper dibromide, $\mathrm{CuBr}_{2}$. Z. Kristallogr. 215, 13-13 (2000).

39. Slot, E., Holst, M. A., van der Zant, H. S. J. \& Zaitsev-Zotov, S. V. One-dimensional conduction in charge-density-wave nanowires. Phys. Rev. Lett. 93, 176602 (2004).

40. Park, C., Kim, S. W. \& Yoon, M. First-principles prediction of new electrides with nontrivial band topology based on one-dimensional building blocks. Phys. Rev. Lett. 120, 026401 (2018).

41. Venkataraman, L., Hong, Y. S. \& Kim, P. Electron transport in a multichannel onedimensional conductor: molybdenum selenide nanowires. Phys. Rev. Lett. 96, 076601 (2006)

42. Zhang, Z. et al. Ultrathin inorganic molecular nanowire based on polyoxometalates. Nat. Commun. 6, 7731 (2015).
43. Hartwig, S. \& Hillebrecht, H. Crystal structures of $\mathrm{NbOI} 3$ and $\mathrm{NbOBr} 3$ - polar double chains in different non-centrosymmetric structures. Z. Anorg. Allg. Chem. 634, 115-120 (2008).

44. Sands, D. E., Zalkin, A. \& Elson, R. E. The crystal structure of NbOCl3. Acta Crystallogr 12, 21-23 (1959).

45. Zhuang, H. L. \& Hennig, R. G. Computational search for single-layer transitionmetal dichalcogenide photocatalysts. J. Phys. Chem. C 117, 20440-20445 (2013).

46. Shulenburger, L., Baczewski, A. D., Zhu, Z., Guan, J. \& Tománek, D. The nature of the interlayer interaction in bulk and few-layer phosphorus. Nano Lett. 15, 8170-8175 (2015).

47. King-Smith, R. D. \& Vanderbilt, D. Theory of polarization of crystalline solids. Phys. Rev. B 47, 1651-1654 (1993).

48. Spaldin, N. A. A beginner's guide to the modern theory of polarization. J. Solid State Chem. 195, 2-10 (2012).

49. Bruyer, E. et al. Possibility of combining ferroelectricity and rashba-like spin splitting in monolayers of the 1T-typetransition-metal dichalcogenides MX2 ( $\mathrm{M}=$ Mo, W; X = S, Se, Te). Phys. Rev. B 94, 195402 (2016).

50. Wan, W., Liu, C., Xiao, W. \& Yao, Y. Promising ferroelectricity in 2D group IV tellurides: a first-principles study. Appl. Phys. Lett. 111, 132904 (2017).

51. Wang, H. \& Qian, X. Two-dimensional multiferroics in monolayer group IV monochalcogenides. 2D Mater. 4, 015042 (2017).

52. Song, W., Fei, R. \& Yang, L. Off-plane polarization ordering in metal chalcogen diphosphates from bulk to monolayer. Phys. Rev. B 96, 235420 (2017).

53. Xu, B. et al. Monolayer Agbip2se6: an atomically thin ferroelectric semiconductor with out-plane polarization. Nanoscale 9, 8427-8434 (2017).

54. Choi, K. J. et al. Enhancement of ferroelectricity in strained $\mathrm{BaTiO} 3$ thin films. Science 306, 1005-1009 (2004).

55. Rouquette, J. et al. Pressure-induced rotation of spontaneous polarization in monoclinic and triclinic PbZr0.52Ti0.48O3. Phys. Rev. B 71, 024112 (2005).

56. Izyumskaya, N. et al. Processing, structure, properties, and applications of PZT thin films. Crit. Rev. Solid State Mater. Sci. 32, 111-202 (2007).

57. Cohen, R. E. Origin of ferroelectricity in perovskite oxides. Nature 358, 136-138 (1992).

58. Ding, W. et al. Prediction of intrinsic two-dimensional ferroelectrics in $\ln 2 \mathrm{Se} 3$ and other III2-VI3 van der Waals materials. Nat. Commun. 8, 14956 (2017).

59. Zhang, Y., Sun, J., Perdew, J. P. \& Wu, X. Comparative first-principles studies of prototypical ferroelectric materials by LDA, GGA, and SCAN meta-GGA. Phys. Rev. B 96, 035143 (2017).

60. Halasyamani, P. S. \& Poeppelmeier, K. R. Noncentrosymmetric oxides. Chem. Mater. 10, 2753-2769 (1998).

61. Olsen, G. H., Aschauer, U., Spaldin, N. A., Selbach, S. M. \& Grande, T. Origin of ferroelectric polarization in tetragonal tungsten-bronze-type oxides. Phys. Rev. B 93, 180101 (2016)

62. Terakura, K. \& Ishibashi, S. Mechanism of covalency-induced electric polarization within the framework of maximally localized Wannier orbitals. Phys. Rev. B 91, 195120 (2015)

63. Zhou, D. et al. Electric field and temperature scaling of polarization reversal in silicon doped hafnium oxide ferroelectric thin films. Acta Mater. 99, 240-246 (2015).

64. Hoffmann, M. et al. Unveiling the double-well energy landscape in a ferroelectric layer. Nature 565, 464-467 (2019).

65. Liu, F. et al. Room-temperature ferroelectricity in CulnP2S6 ultrathin flakes. Nat. Commun. 7, 12357 (2016).

66. Xiao, J. et al. Intrinsic two-dimensional ferroelectricity with dipole locking. Phys. Rev. Lett. 120, 227601 (2018).

67. Kresse, G. \& Furthmüller, J. Efficiency of ab-initio total energy calculations for metals and semiconductors using a plane-wave basis set. Comput. Mater. Sci. 6 , 15-50 (1996).

68. Kresse, G. \& Furthmüller, J. Efficient iterative schemes for ab initio totalenergy calculations using a plane-wave basis set. Phys. Rev. B 54, 11169-11186 (1996).

69. Kresse, G. \& Hafner, J. Ab initio molecular-dynamics simulation of the liquidmetal-amorphous-semiconductor transition in germanium. Phys. Rev. B 49, 14251-14269 (1994).

70. Kresse, G. \& Joubert, D. From ultrasoft pseudopotentials to the projector augmented-wave method. Phys. Rev. B 59, 1758-1775 (1999).

71. Perdew, J. P., Ernzerhof, M. \& Burke, K. Rationale for mixing exact exchange with density functional approximations. J. Chem. Phys. 105, 9982-9985 (1996).

72. Perdew, J. P., Burke, K. \& Ernzerhof, M. Generalized gradient approximation made simple. Phys. Rev. Lett. 77, 3865 (1996).

73. Grimme, S. Semiempirical Gga-type density functional constructed with a longrange dispersion correction. J. Comput. Chem. 27, 1787-1799 (2006).

74. Krukau, A. V., Vydrov, O. A., Izmaylov, A. F. \& Scuseria, G. E. Influence of the exchange screening parameter on the performance of screened hybrid functionals. J. Chem. Phys. 125, 224106 (2006). 
75. Togo, A. \& Tanaka, I. First principles phonon calculations in materials science. Scr. Mater. 108, 1-5 (2015).

76. Mills, G., Jónsson, H. \& Schenter, G. K. Reversible work transition state theory: application to dissociative adsorption of hydrogen. Surf. Sci. 324, 305-337 (1995)

77. Evans, D. J. Computer "experiment" for nonlinear thermodynamics of Couette flow. J. Chem. Phys. 78, 3297-3302 (1983)

78. Hoover, W. G., Ladd, A. J. C. \& Moran, B. High-strain-rate plastic flow studied via nonequilibrium molecular dynamics. Phys. Rev. Lett. 48, 1818-1820 (1982).

\section{ACKNOWLEDGEMENTS}

We acknowledge generous grants of high-performance computing resources provided by $\mathrm{NCl}$ National Facility and the Pawsey Supercomputing Centre through the National Computational Merit Allocation Scheme supported by the Australian Government and the Government of Western Australia. A.D. also greatly appreciates the financial support of the Australian Research Council under Discovery Project (DP170103598, DP210100721, and DP210100331). S.S. acknowledges financial support from the Irish Research Council [IR- CLA/2019/127].

\section{AUTHOR CONTRIBUTIONS}

L.Z. performed the conceptualization, investigation, and writing-original draft. C.T., S.S., and A.D. discussed the results and provided many suggestions. A.D. also performed the supervision and writing-review and editing.

\section{COMPETING INTERESTS}

The authors declare no competing interests.

\section{ADDITIONAL INFORMATION}

Supplementary information The online version contains supplementary material available at https://doi.org/10.1038/s41524-021-00602-9.

Correspondence and requests for materials should be addressed to A.D.

Reprints and permission information is available at http://www.nature.com/ reprints

Publisher's note Springer Nature remains neutral with regard to jurisdictional claims in published maps and institutional affiliations.

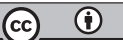

Open Access This article is licensed under a Creative Commons Attribution 4.0 International License, which permits use, sharing, adaptation, distribution and reproduction in any medium or format, as long as you give appropriate credit to the original author(s) and the source, provide a link to the Creative Commons license, and indicate if changes were made. The images or other third party material in this article are included in the article's Creative Commons license, unless indicated otherwise in a credit line to the material. If material is not included in the article's Creative Commons license and your intended use is not permitted by statutory regulation or exceeds the permitted use, you will need to obtain permission directly from the copyright holder. To view a copy of this license, visit http://creativecommons. org/licenses/by/4.0/.

(c) The Author(s) 2021 